\title{
ON OPTIMAL INTERCONNECTIONS FOR VLSI
}




\title{
THE KLUWER INTERNATIONAL SERIES IN ENGINEERING AND COMPUTER SCIENCE
}

\author{
VLSI, COMPUTER ARCHITECTURE AND \\ DIGITAL SIGNAL PROCESSING \\ Consulting Editor \\ Jonathan Allen
}

Other books in the series:

MIXED-MODE SIMULATION AND ANALOG MULTILEVEL SIMULATION, Resve Saleh, Shyh-Jou, A. Richard Newton

ISBN: 0-7923-9473-9

CAD FRAMEWORKS: Principles and Architectures, Pieter van der Wolf ISBN: 0-7923-9501-8

PIPELINED ADAPTIVE DIGITAL FILTERS, Naresh R. Shanbhag, Keshab K. Parhi ISBN: 0-7923-9463-1

TIMED BOOLEAN FUNCTIONS: A Unified Formalism for Exact Timing Analysis, William K.C. Lam, Robert K. Brayton

ISBN: 0-7923-9454-2

AN ANALOG VLSI SYSTEM FOR STEREOSCIPIC VISION, Misha Mahowald ISBN: 0-7923-944-5

ANALOG DEVICE-LEVEL LAYOUT AUTOMATION, John M. Cohn, David J. Garrod, Rob A. Rutenbar, L. Richard Carley

ISBN: 0-7923-9431-3

VLSI DESIGN METHODOLOGIES FOR DIGITAL SIGNAL PROCESSING

ARCHITECTURES, Magdy A. Bayoumi ISBN: 0-7923-9428-3

CIRCUIT SYNTHESIS WITH VHDL, Roland Airiau, Jean-Michel Berge, Vincent Olive ISBN: 0-7923-9429-1

ASYMPTOTIC WAVEFORM EVALUATION, Eli Chiprout, Michel S. Nakhla ISBN: 0-7923-9413-5

WAVE PIPELINING: THEORY AND CMOS IMPLEMENTATION,

C. Thomas Gray, Wentai Liu, Ralph K. Cavin, III ISBN: 0-7923-9398-8

CONNECTIONIST SPEECH RECOGNITION: A Hybrid Appoach, H. Bourlard, N. Morgan ISBN: 0-7923-9396-1

BiCMOS TECHNOLOGY AND APPLICATIONS, SECOND EDITION, A.R. Alvarez ISBN: 0-7923-9384-8

TECHNOLOGY CAD-COMPUTER SIMULATION OF IC PROCESSES AND DEVICES,

R. Dutton, Z. Yu

ISBN: 0-7923-9379

VHDL 92, THE NEW FEATURES OF THE VHDL HARDWARE DESCRIPTION LANGUAGE, J. Bergé, A. Fonkoua, S. Maginot, J. Rouillard ISBN: 0-7923-9356-2

APPLICATION DRIVEN SYNTHESIS, F. Catthoor, L. Svenson ISBN:0-7923-9355-4

ALGORITHMS FOR SYNTHESIS AND TESTING OF ASYNCHRONOUS CIRCUITS, L. Lavagno, A. Sangiovanni-Vincentelli ISBN: 0-7923-9364-3

HOT-CARRIER RELIABILITY OF MOS VLSI CIRCUITS, Y. Leblebici, S. Kang ISBN: $0-7923-9352-\mathrm{X}$ 


\title{
ON OPTIMAL INTERCONNECTIONS FOR VLSI
}

\author{
Andrew B. Kahng \\ University of California/Los Angeles
}

Gabriel Robins

University of Virginia 


\section{Library of Congress Cataloging-in-Publication}

A C.I.P. Catalogue record for this book is available from the Library of Congress.

ISBN 978-1-4419-5145-8

DOI 10.1007/978-1-4757-2363-2

ISBN 978-1-4757-2363-2 (eBook)

Copyright $(1995$ by Springer Science+Business Media New York

Originally published by Kluwer Academic Publishers in 1995

Softcover reprint of the hardcover 1st edition 1995

Fourth Printing 2001

This printing is a digital duplication of the original edition.

All rights reserved. No part of this publication may be reproduced, stored in a retrieval system or transmitted in any form or by any means, mechanical, photo-copying, recording, or otherwise, without the prior written permission of the publisher, Springer Science+Business Media, LLC.

Printed on acid-free paper. 
To the field of VLSI CAD 


\section{CONTENTS}

LIST OF FIGURES xi

LIST OF TABLES xv

1 PRELIMINARIES 1

1.1 Preface 1

1.2 The Domain of Discourse: Routing in VLSI Physical Design 2

1.3 Overview of the Book 8

1.3.1 Minimum Area: The Steiner Minimal Tree Problem 8

1.3.2 Minimum Delay: Toward Optimal-Delay Routing Trees 9

1.3.3 Minimum Skew: The Zero-Skew Clock Routing Problem 11

1.3.4 Multiple Objectives $\quad 12$

1.4 Acknowledgments 13

2 AREA 16

2.1 Introduction 17

2.2 Performance Bounds for MST-Based Strategies 25

2.2.1 Counterexamples in Two Dimensions 25

2.2.2 Counterexamples in Higher Dimensions 30

2.3 Iterated 1-Steiner (IIS) 31

2.3.1 Finding 1-Steiner Points Efficiently 33

2.3.2 The I1S Performance Ratio 34

2.3.3 The Method of Zelikovsky 41

2.4 Enhancing I1S Performance 43

2.4.1 A Batched Variant $\quad 43$

2.4.2 A Perturbative Variant $\quad 46$

2.4.3 Parallel Implementation $\quad 48$ 
2.5 Practical Implementation Options for I1S 48

2.5.1 Incremental MST Updates in Batched 1-Steiner 48

2.5.2 MST Degree Bounds $\quad 50$

2.6 On The Maximum MST Degree 54

2.7 Steiner Trees in Graphs 56

2.8 Experimental Results $\quad 59$

3 DELAY 64

3.1 Preliminaries 65

3.1.1 Definitions 66

3.1.2 The Linear and Elmore Delay Approximations $\quad 67$

3.2 Geometric Approaches to Delay Minimization 69

$\begin{array}{ll}\text { 3.2.1 Early Cost-Radius Tradeoffs } & 70\end{array}$

The Bounded-Prim (BPRIM) Algorithm $\quad 72$

Extensions of BPRIM $\quad 74$

$\begin{array}{ll}\text { 3.2.2 Shallow-Light Constructions } & 76\end{array}$

$\begin{array}{ll}\text { The BRBC Algorithm } & 79\end{array}$

Bounded-Radius Steiner Trees $\quad 81$

Improvements in Geometry $\quad 83$

Sink-Dependent Bounds and the Shallow-Light Result $\quad 84$

The KRY Algorithm $\quad 86$

3.2.3 The Prim-Dijkstra Tradeoff $\quad 88$

The PD1 Tradeoff $\quad 88$

The PD2 Tradeoff $\quad 90$

3.2.4 Rectilinear Steiner Arborescences $\quad 91$

3.2.5 Experimental Results and Discussion 96

Comparison of Cost-Radius Tradeoffs 96

Comparison of Signal Delays $\quad 98$

Steiner Routing 100

3.3 Minimization of Actual Delay 103

3.3.1 Greedy Optimization of Elmore Delay 103

3.3.2 The Critical-Sink Routing Tree Problem 105

Geometric CSRT Heuristics 108

CSRT Heuristics That Optimize Elmore Delay Directly 113

3.3.3 Experimental Results $\quad 115$

CS-Steiner Trees 115 
3.3.4 Optimal-Delay Routing Trees 120

Spanning Trees and BBORT 121

Toward Elmore Delay-Optimal Steiner Trees 123

Steiner Trees and BB-SORT-C 126

$\begin{array}{ll}\text { 3.3.5 Remarks } & 127\end{array}$

$\begin{array}{lll}3.4 \text { New Directions } & 128\end{array}$

3.4.1 Wiresizing 129

3.4.2 Non-Tree Routing 134

4 SKEW 140

4.1 Preliminaries 141

4.2 An Early Matching-Based Approach 145

4.2.1 Pathlength-Balanced Trees 146

4.2.2 The Iterated Matching Approach 147

4.2.3 Extension to Building-Block Design 152

4.2.4 Empirical Tests $\quad 155$

Results for Cell-Based Designs $\quad 155$

Results for Building-Block Designs 159

Remarks 161

4.3 DME: Exact Zero Skew With Minimum Wirelength 163

4.3.1 Bottom-Up Phase: The Tree of Merging Segments 165

4.3.2 Top-Down Phase: Embedding of Nodes 169

4.3.3 Application of DME to Linear Delay 170

Calculating Edge Lengths $\quad 170$

Optimality of DME for Linear Delay 172

4.3.4 Application to Elmore Delay 176

Calculating Edge Lengths in the Elmore Delay Model $\quad 176$

Suboptimality of DME for Elmore Delay 178

4.3.5 Experimental Results and Discussion $\quad 179$

Results for the Linear Delay Model 180

Results for the Elmore Delay Model 180

Remarks 183

4.4 Planar-Embeddable Trees 184

4.4.1 Single-Pass DME $\quad 187$

4.4.2 The Planar-DME Algorithm 188 
4.4.3 Experimental Results and Discussion 192

$\begin{array}{lll}4.5 & \text { Remarks } & 193\end{array}$

5 MULTIPLE OBJECTIVES 197

$\begin{array}{ll}5.1 \text { Minimum Density Trees } & 198\end{array}$

5.1.1 Heuristics for Minimum Density Trees 200

The COMB Construction 200

A Chain-Peeling Method 202

5.1.2 Performance Bounds 204

Density Bounds 204

Cost Bounds 208

5.1.3 Triple Optimization $\quad 210$

Minimizing Skew, Density, and Total Wirelength 210

Minimizing Radius, Density, and Total Wirelength 212

5.1.4 Experimental Results $\quad 213$

$\begin{array}{lll}5.2 & \text { Multi-Weighted Graphs } & 215\end{array}$

5.3 Prescribed-Width Routing 223

5.3.1 Prescribed-Width Routing by Network Flows 224

Problem Formulation $\quad 225$

A Network Flow Based Approach $\quad 229$

A Test Implementation $\quad 234$

5.3.2 Simulation Results $\quad 235$

A APPENDIX: SIGNAL DELAY ESTIMATORS 239

A.1 Basics 239

A.1.1 Elmore Delay $\quad 241$

A.1.2 Two-Pole Analysis $\quad 242$

$\begin{array}{lll}\text { A.2 Accuracy and Fidelity } & 246\end{array}$

$\begin{array}{ll}\text { A.2.1 Accuracy } & 247\end{array}$

$\begin{array}{ll}\text { A.2.2 Fidelity } & 248\end{array}$

REFERENCES 252

$\begin{array}{ll}\text { AUTHOR INDEX } & 275\end{array}$

$\begin{array}{ll}\text { TERM INDEX } & 281\end{array}$ 


\section{LIST OF FIGURES}

\section{Chapter 1}

1.1 The VLSI design process. 3

1.2 A channel intersection graph. 5

\section{Chapter 2}

2.1 An MST and an SMT for the same pointset. 18

2.2 Hanan's theorem. 19

2.3 Two types of SMTs. 20

2.4 Cost of the tour is equal to the bounding box perimeter. 22

2.5 Optimal overlap of MST edges within their bounding boxes. 26

2.6 Example with $\frac{\operatorname{cost}(M S T-\text { Overlap })}{\operatorname{cost}(S M T)}=\frac{3}{2} . \quad 27$

2.7 A separable MST where $\frac{\operatorname{cost}(M S T-\text { Overlap })}{\operatorname{cost}(S M T)}$ is close to $\frac{3}{2}$. 28

2.8 The class $C$ of greedy Steiner tree heuristics. 29

2.9 Example forcing a performance ratio arbitrarily close to $\frac{5}{3}$. 31

2.10 The Iterated 1-Steiner (I1S) algorithm. 32

2.11 Execution of Iterated 1-Steiner. 32

2.12 Dirichlet cells with respect to directions $\theta_{1}$ and $\theta_{2}$. 33

2.13 Locally replacing each plus with an MST. 37

$2.14 \mathrm{I} 1 \mathrm{~S}$ achieves $\frac{1}{3}$ of the maximum possible savings. 38

2.15 The two possible Steiner tree topologies on 4 points. 38

2.16 Example where the I1S performance ratio is $\frac{7}{6}$. 38

2.17 Example where the I1S performance ratio is $\frac{13}{11}$. 39

2.18 Example where I1S outperforms MST-Overlap. $\quad 39$

2.19 The construction of Berman et al.. 40

2.20 Batching computations within the 1-Steiner approach. 45

2.21 The Batched 1-Steiner (B1S) algorithm. 45

2.22 The Perturbative Iterated $k$-Steiner (PIkS) method. 47 
2.23 Dynamic MST maintenance. $\quad 49$

2.24 Linear-time dynamic MST maintenance. $\quad 50$

2.25 The diagonal partition of the plane. 51

2.26 A truncated cube induces a cuboctahedral space partition. $\quad 53$

2.27 The KMB heuristic for the GSMT problem. 57

2.28 The Graph Iterated 1-Steiner algorithm. 58

2.29 Example of the output of B1S on 300 points. $\quad 60$

2.30 Average performance and speed of B1S. 62

2.31 Average performance of PI2S, B1S, and OPT. 63

\section{Chapter 3}

3.1 Example with SPT cost $\Omega(|N|)$ times the MST cost. 71

3.2 Increasing $\epsilon$ may decrease tree cost but increase the radius. $\quad 71$

$\begin{array}{lll}3.3 & \text { The BPRIM algorithm. } & 73\end{array}$

$\begin{array}{ll}3.4 & \text { BPRIM radius can be arbitrarily large. }\end{array}$

$\begin{array}{lll}3.5 & \text { BPRIM has unbounded cost performance ratio for any } \epsilon \text {. } & 75\end{array}$

$\begin{array}{lll}3.6 & \text { A more general BPRIM template. } & 75\end{array}$

3.7 Unbounded cost performance ratio for $\mathrm{H} 2$ and $\mathrm{H} 3$. 76

3.8 Example for which BPRIM outperforms variants $\mathrm{H} 2$ and $\mathrm{H} 3$. $\quad 77$

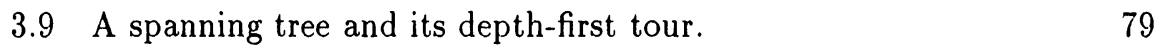

3.10 The BRBC algorithm. $\quad 80$

3.11 The BRBC construction. $\quad 81$

3.12 The KRY algorithm. 87

3.13 Sample executions for PD1 and PD2. 89

3.14 A minimum-cost rectilinear Steiner arborescence. 92

3.15 Illustration of the RSA heuristic of Rao et al. 93

3.16 Safe moves in the heuristic RSA construction. 94

3.17 A pathological instance for existing RSA heuristics. $\quad 95$

3.18 The BPRIM and BRBC cost-radius tradeoffs. 97

3.19 Graph of radius ratio $\left(\frac{r(T)}{r\left(T_{S}\right)}\right)$ versus cost ratio $\left(\frac{\cos t(T)}{\cos t\left(T_{M}\right)}\right) \quad 99$

3.20 Execution of PD1 with $c=0.5$. 101

3.21 The ERT Algorithm. 104

3.22 Example of the progressive SERT Steiner tree construction. 106

3.23 Effect of the CSRT formulation on the optimal solution. 109

3.24 The CSRT problem is NP-hard for any technology parameters. 110 
3.25 The CS-Steiner heuristic.

3.26 Removal of $V$ and $U$ configurations by GSR. 111

3.27 Pseudo-code for Global Slack Removal. 112

3.28 The SERT-C Algorithm. 114

3.29 SERT-C tree constructions for an 8-sink net. 116

3.30 Branch-and-Bound Optimal Routing Tree algorithm. 121

3.31 Maximal segment $M$ and its four branches. 125

3.32 Counterexample to the separability property. 131

3.33 The Static Greedy Wiresizing algorithm. 132

3.34 The DWSERT algorithm. 133

$\begin{array}{ll}3.35 \text { Comparison of different wiresizing constructions. } & 135\end{array}$

3.36 Adding an edge to the MST reduces maximum sink delay. $\quad 136$

3.37 The Low Delay Routing Graph algorithm. 137

$\begin{array}{ll}3.38 \text { Empirical results for the LDRG heuristic. } & 138\end{array}$

\section{Chapter 4}

4.1 Two bad clock trees. 147

4.2 An optimal geometric matching over four terminals. 148

4.3 CLOCK1: pathlength-balanced tree heuristic. 149

4.4 An example execution of CLOCK1 on a set of 16 terminals. 150

4.5 H-flipping to reduce pathlength skew. 151

4.6 An edge belongs to at most one shortest path in a matching. 153

4.7 CLOCK2: pathlength-balanced tree heuristic. 155

4.8 An example execution of CLOCK2. 156

4.9 Output of variant $\mathrm{GR}+\mathrm{E}+\mathrm{H}$ on the Primary2 layout. 161

4.10 Further optimizations can use loci of balance points. 163

4.11 A TRR with core and radius as indicated. 166

4.12 Construction of a merging segment: two cases. $\quad 167$

4.13 Example of a tree of merging segments. 167

4.14 Intersecting two TRRs after 45-degree rotation. 168

4.15 Construction of the tree of merging segments. 169

4.16 Procedure Find_Exact_Placements. 170

4.17 Construction of the ZST by top-down embedding. 171

4.18 Optimal placement of siblings must satisfy distance constraint. 175

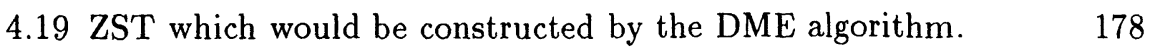


4.20 Output of KCR+DME on the Primary2 benchmark layout. 182

4.21 Edges of an optimal planar ZST may overlap. $\quad 185$

4.22 Contrast between the H-tree and Zhu-Dai solutions. 186

4.23 Rules to choose embedding point and splitting line. 190

4.24 The Planar-DME Algorithm. 193

4.25 An example of Planar-DME execution. 194

4.26 Planar-DME and Zhu-Dai ZSTs for Primary2 benchmark. 196

\section{Chapter 5}

5.1 A four-terminal signal net. 199

5.2 A minimum density tree for a signal net. 200

5.3 Execution of the COMB construction. 201

5.4 Algorithm COMB for minimum-density spanning trees. 201

5.5 Execution of the COMB_ST Steiner tree construction. 202

5.6 Algorithm COMB_ST: for minimum-density Steiner trees. 202

5.7 Algorithm PEEL for low-density trees. 203

5.8 A class of worst-case examples for PEEL. 203

5.9 Expected minimum density of a net. 206

5.10 Computing a non-uniform lower bound on density. 206

5.11 Combining chains into a low-density tree. 208

5.12 Partitioning a net into strips/chains. 211

5.13 A 2-weighted graph and its induced graphs. 217

5.14 MST cost on multi-weighted graphs has no upper bound. 219

5.15 An upper bound for metric multi-weighted graphs. 220

5.16 A tighter upper bound for 3-terminal nets. 221

5.17 Topology of the three spanning trees. 222

5.18 A path $P$ between two points $s \in S$ and $t \in T$. 226

5.19 A $d$-separating path $\bar{P}$. 227

5.20 A discretized representation of a region. 228

5.21 A node and its $d$-neighborhood. 231

5.22 Transformation of PWP into network flow. 232

5.23 Transformation into an arc-capacitated flow network. 233

5.24 Finding a minimum cost prescribed-width path. 234

5.25 Prescribed-width paths among polygonal obstacles. 237

5.26 Prescribed-width path in a random smooth region. 238 


\section{LIST OF TABLES}

\section{Chapter 1}

\section{Chapter 2}

\section{Chapter 3}

3.1 Interconnect technology parameters. $\quad 69$

3.2 Equivalences of algorithm parameters. 98

3.3 Average source-sink delay in spanning constructions. 100

3.4 Average source-sink delay in Steiner constructions. 102

$\begin{array}{lll}3.5 & \text { CS-Steiner results. } & 117\end{array}$

3.6 ERT, SERT and SERT-C results for 5-terminal nets. 118

3.7 ERT, SERT and SERT-C results for 9-terminal nets. 119

3.8 Near-optimality of ERT delay and tree cost. 122

3.9 Near-optimality of SERT-C delay and tree cost. 127

3.10 Performance comparisons for the DWSERT algorithm. 134

\section{Chapter 4}

4.1 Average clock tree cost for the various heuristics. 158

4.2 Average clock tree cost for the various heuristics (continued). 158

4.3 Average pathlength skew for the various heuristics. 159

4.4 Average pathlength skew for the various heuristics (continued). 159

4.5 Min, ave, and max tree cost for MMM and GR+E+H. 160

4.6 Min, ave, and max pathlength skew for MMM and GR+E+H. 160

4.7 Average tree costs and skews of KMB and CLOCK2 trees. 162

4.8 Delay and capacitance at each internal node. 180

4.9 Effect of DME on KCR and BB using linear delay. 181

4.10 Comparison of algorithms for the Elmore delay model. 181 
4.11 Comparison of Planar-DME with other algorithms.

\section{Chapter 5}

5.1 Tree density statistics.

5.2 Tree cost statistics.

\section{Appendix A}

A.1 Accuracy of the Linear, Elmore and Two-Pole estimates.

A.2 Fidelity: average difference in rankings of topologies.

A.3 Average SPICE delay ratios for the top 19 topologies.

A.4 SPICE suboptimality of Elmore delay (percent). 


\section{ON OPTIMAL INTERCONNECTIONS FOR VLSI}

